\title{
Tumor Antigen
}

National Cancer Institute

\section{Source}

National Cancer Institute. Tumor Antigen. NCI Thesaurus. Code C912.

1. antigens that may be frequently associated with tumors or may be specifically found on tumor cells of the same orig in (tumor specific); 2. tumor antigens may also be associated with replication and transformation by certain DNA tumor viruses, including adenoviruses and papovaviruses. Neoantigens. Any immunologically detectable marker whose presence or amount indicates the presence of a tumor. 